\title{
Analysis of mutation of the c-Kit gene and PDGFRA in gastrointestinal stromal tumors
}

\author{
CHUN-WEI XU ${ }^{1 *}$, SHAN LIN $^{2 *}$, WU-LONG WANG ${ }^{3}$, WEN-BIN GAO ${ }^{2}$, JIN-YAN LV ${ }^{2}$, JING-SHAN GAO ${ }^{4}$, \\ LI-YING ZHANG ${ }^{1}$, YANG LI ${ }^{5}$, LIN WANG ${ }^{6}$, YU-PING ZHANG ${ }^{7}$ and YU-WANG TIAN ${ }^{1}$ \\ ${ }^{1}$ Department of Pathology, The General Military Hospital of Beijing PLA, Beijing 100700; ${ }^{2}$ Department of Oncology, \\ Affiliated Zhongshan Hospital of Dalian University, Dalian, Liaoning 116001; ${ }^{3}$ Department of Oncology, \\ The Second Affiliated Hospital of Baotou Medical College, Baotou, Inner Mongolia 014030; \\ ${ }^{4}$ Department of Clinical Medicine, Dalian Medical University, Dalian, Liaoning 116044; ${ }^{5}$ Department of Oncology, \\ The General Military Hospital of Beijing PLA, Beijing 100700; ${ }^{6}$ Department of Pathology, Shanxi Da Hospital, \\ Shanxi Academy of Medical Sciences, Taiyuan, Shanxi 030001; 7 Department of Pathology, \\ The People's Hospital of Weifang, Weifang, Shandong 261041, P.R. China
}

Received October 29, 2014; Accepted May 18, 2015

DOI: 10.3892/etm.2015.2613

\begin{abstract}
The aim of the present study was to investigate mutation status of the c-Kit gene (KIT) and PDGFRA in patients with a gastrointestinal stromal tumor (GIST). In total, 93 patients with a GIST were included in the study, in which polymerase chain reaction amplification and gene sequencing were used to detect the sequences of exons 9, 11, 13 and 17 in KIT and exons 12 and 18 in PDGFRA. KIT mutations were detected in 64 cases $(68.82 \%)$, of which exon 11 mutations were detected in 56 cases $(60.22 \%)$, exon 13 mutations were detected in three cases $(3.23 \%)$ and one case $(1.08 \%)$ was shown to have a mutation in exon 17. The most common mutation in exon 11 was a deletion, which accounted for $55.36 \%$ (31/56) of the cases, followed by a point mutation observed in $26.79 \%$ (15/56) of the cases, while an insertion (tandem repeats) was identified in $14.29 \%(8 / 56)$ of the cases, and $3.57 \%(2 / 56)$ of the exon 11 mutations were deletions associated with a point mutation. The majority of the mutations were heterozygous, with only a few homozygous mutations. Mutational analysis revealed the mutations to be more concentrated in the classic hot zone at the 5'-end, followed by the tandem repeat frame at the 3 '-end. In four cases, a mutation was detected in exon 18 of PDGFRA, of which one was associated with a mutation in KIT. The remaining three cases $(10.34 \%$, $3 / 29$ ) were not associated with mutations in KIT and accounted
\end{abstract}

Correspondence to: Professor Wen-Bin Gao, Department of Oncology, Affiliated Zhongshan Hospital of Dalian University, 6 Jiefang Street, Dalian, Liaoning 116001, P.R. China

E-mail: drwenbingao@163.com

*Contributed equally

Key words: gastrointestinal stromal tumors, c-Kit, PDGFRA, gene mutation, molecular detection for 37.5\% (3/8) of the CD117-negative GIST cases. Therefore, the majority of the GIST cases were characterized by mutations in KIT or PDGFRA, which were directly associated with the disease. Pairs of different mutations in the same exon of $K I T$, or KIT mutations coupled with pairs of mutations in PDGFRA, were detected in a small number of patients. Imatinib is a small molecule tyrosine kinase inhibitor and is the first line targeted treatment for GIST, resulting in markedly improved survival rates. Thus, gene mutation genotyping may provide inspiration and guidance for imatinib-based targeted cancer therapy.

\section{Introduction}

Gastrointestinal stromal tumors (GISTs) are the most common type of primary gastrointestinal mesenchymal tumor, accounting for $2 \%$ of all gastrointestinal tumors. GISTs are typically identified via immunohistochemical staining for CD117 in the neoplastic cell membrane, which is generally positive, and in neoplastic cytoplasm or nucleus, which is diffusely positive, and exhibit a phenotype similar to that of interstitial cell of Cajal. The majority of GIST cases exhibit an activated c-Kit gene (KIT) or PDGFRA mutations, which obtain conductivity activation independently of each other $(1,2)$. In the absence of ligand binding, c-Kit or PDGFRA proteins maintain a sustained tyrosine kinase activity, which activates downstream signaling pathways (1-3).

With regard to GIST, previous studies investigating the associations among the mutation rate, mutation types and biological behavior in KIT or PDGFRA are not in agreement (4-7). Imatinib is a targeted drug therapy for limiting the pathogenesis of the GIST, and has been widely adopted for the treatment of GISTs in recent years. In vitro and in vivo experiments have clinically demonstrated that the efficacy of imatinib against GIST depends considerably on the presence or absence of genetic mutations, and on the mutation sites and types $(8,9)$. Therefore, investigations into the mutations associated with GIST have high clinical significance. 
In the present study, polymerase chain reaction (PCR) amplification and sequencing methods were used to detect the presence of gene mutations and analyze GIST-associated gene mutations in patients from North China. The aim of the current study was to provide the basis of the molecular pathology underlying GIST to allow for targeted therapy with imatinib.

\section{Materials and methods}

Specimen collection. Test specimens were collected from the General Military Hospital of Beijing PLA (Beijing, China), the Affiliated Zhongshan Hospital of Dalian University (Dalian, China) and the People's Hospital of Weifang (Weifang, China) between 2007 and 2013 for the pathological diagnosis of GIST. Every specimen was reviewed by three pathologists, who collected the relevant clinical data. All protocols were approved by the Human Clinical and Research Ethics Committees of the General Military Hospital of Beijing PLA (Beijing, China), the Affiliated Zhongshan Hospital of Dalian University (Dalian, China) and the People's Hospital of Weifang (Weifang, China). Written informed consent was obtained from all patients.

Reagents and instruments. A DNA extraction kit was purchased from Qiagen (Hilden, Germany) and a nucleic acid-protein concentration measuring instrument was purchased from Shanghai Chong Meng Biotechnology Co., Ltd. (B-500; Shanghai, China). CD117 (clone YR145), S-100 (clone 4C4.9) and DOG-1 (clone SP31) antibodies were purchased from Fuzhou Maixin Biotechnology Development Co., Ltd. (Fuzhou, China), while smooth muscle actin (SMA; clone IA4) and desmin (clone ZC18) antibodies were purchased from Beijing Zhongshan Golden Bridge Biotechnology Co., Ltd. (Beijing, China). Primers synthesis was performed by Shanghai Handsome Biotech Co., Ltd. (Shanghai, China), and the sequences are shown in Table I. An ABI 9700 PCR Cycler was purchased from Applied Biosystems Life Technologies (Foster City, CA, USA).

Immunohistochemistry. All specimens (4- $\mu \mathrm{m}$ serial sections) were embedded in paraffin and incubated at $60^{\circ} \mathrm{C}$ for $90 \mathrm{~min}$. Using the EnVision two-step method, the experiments were performed in strict accordance with the kit instructions. Tissue sections were deparaffinized, rehydrated and incubated with $3 \% \mathrm{H}_{2} \mathrm{O}_{2}$ in methanol for $15 \mathrm{~min}$ at room temperature to eliminate endogenous peroxidase activity. The antigen was retrieved at $95^{\circ} \mathrm{C}$ for $20 \mathrm{~min}$ by placing the slides in $0.01 \mathrm{M}$ sodium citrate buffer ( $\mathrm{pH}$ 6.0). Slides were then incubated with primary antibody at $4^{\circ} \mathrm{C}$ overnight. After incubation at room temperature for $30 \mathrm{~min}$ with biotinylated secondary antibody, slides were incubated with streptavidin-peroxidase complex at room temperature for $30 \mathrm{~min}$. Then slides were immunostained with 3, 3'-diaminobenzidine, and counterstained with Mayer's hematoxylin (10).

GIST diagnostic criteria. Among the 93 specimens collected, 85 cases were morphologically consistent with a diagnosis of GIST, since CD117 immunohistochemistry revealed strongly positive diffusion in the cellular membranes. Among the remaining eight CD117-negative cases, three cases were found to be positive for DOG-1. Although the remaining five cases were CD117-negative, due to the negativity for SMA, desmin and S-100, and following the exclusion of neurogenic tumors and of smooth muscle origin, the cases were included in the study since they were morphologically consistent with a GIST.

Primerdesign.KIT and PDGFRA gene sequences were obtained from the GenBank database (http://www.ncbi.nlm.nih.gov) under the accession numbers of NC_000004 and NG_009250, respectively. Primers were designed using OLIGO Primer analysis software (Molecular Biology Insights, Inc., Colorado Springs, CO, USA). Attention was paid to prevent the formation of complementary sequences over three base pairs (bp) or between primers. The primer fragment size ranged between 190 and $270 \mathrm{bp}$, while the length of the primer ranged between 17 and $25 \mathrm{bp}$. The GC content of the primers varied between 40 and $70 \%$, and the melting temperatures of the forward and reverse primers were maintained as similar as possible. The primer products were checked with BLAST (http://blast.ncbi.nlm.nih.gov/Blast.cgi), with all the primer products meeting the requirements. Primer synthesis was conducted by the Shanghai Handsome Biotech Co., Ltd.

DNA extraction. DNA was extracted from the various tissue specimens using the QIAamp DNA FFPE Tissue kit, according to the manufacturer's instructions. For each sample, five sections of paraffin-embedded tissues of $10-\mu \mathrm{m}$ thickness were used for DNA extraction. Following isolation, the purity and concentration of the DNA were estimated. DNA concentration was determined using a NanoDrop spectrophotometer, purchased from (Thermo Fisher Scientific, Waltham, MA, USA). A 1-ml sample of water was used as a blank control to zero the spectrophotometer. The aborbance of 5- $\mu \mathrm{l}$ samples dissolved in $1 \mathrm{ml}$ water was measured at 280 and $260 \mathrm{~nm}$. A ratio of $>1.8$ was considered to indicate that the DNA purity and concentration was qualified.

PCR amplification. PCR assays were conducted in a 50- $\mu 1$ reaction system, which contained $25 \mu 12 \mathrm{X}$ Taq PCR Master Mix, $2 \mu$ l DNA template, $2 \mu 1$ (10 pmol) each primer and $19 \mu 1$ sterile deionized water. The reaction conditions were as follows: Initial denaturation at $94^{\circ} \mathrm{C}$ for $5 \mathrm{~min}$, followed by 45 cycles of annealing at $94^{\circ} \mathrm{C}$ for $30 \mathrm{sec}, 56^{\circ} \mathrm{C}$ for $45 \mathrm{sec}$ and $72^{\circ} \mathrm{C}$ for $1 \mathrm{~min}$, and a final extension at $72^{\circ} \mathrm{C}$ for $5 \mathrm{~min}$, after which the samples were cooled to $4^{\circ} \mathrm{C}$ for $5 \mathrm{~min}$. Sterile deionized water was used instead of the template as the negative control. The purified PCR products were sequenced by Beijing Jin Wei Zhi Biotech Co., Ltd. (Beijing, China). Following the identification of the mutations, specimens carrying mutations were reverse-sequenced for confirmation.

Statistical analysis. Data were analyzed using SPSS software, version 19.0 (IBM SPSS, Armonk, NY, USA). Results were analyzed using $\chi^{2}$ and Fisher's exact tests, with a test level of $\alpha=0.05$. The P-value was set to bilateral distribution, and $\mathrm{P}<0.05$ was considered to indicate a statistically significant difference.

\section{Results}

Clinical and pathological features of GIST. Among the 93 patients pathologically confirmed to have a GIST, 53 were 
Table I. Primers for each exon of KIT and PDGFRA.

\begin{tabular}{|c|c|c|}
\hline Exon & Primer sequences & Fragment length (bp) \\
\hline $9(K I T)$ & $\begin{array}{l}\text { Forward: TCCTAGAGTAAGCCAGGGCTT } \\
\text { Reverse: TGGTAGACAGAGCCTAAACATCC }\end{array}$ & 261 \\
\hline $11($ KIT) & $\begin{array}{l}\text { Forward: CCAGAGTGCTCTAATGACTG } \\
\text { Reverse: TGACATGGAAAGCCCCTGTT }\end{array}$ & 225 \\
\hline $13(K I T)$ & $\begin{array}{l}\text { Forward: GCTTGACATCAGTTTGCCAG } \\
\text { Reverse: AAAGGCAGCTTGGACACGCCTTTA }\end{array}$ & 193 \\
\hline $17(K I T)$ & $\begin{array}{l}\text { Forward: TACAAGTTAAAATGAATTTAAATGGT } \\
\text { Reverse: AAGTTGAAACTAAAAATCCTTTGC }\end{array}$ & 228 \\
\hline $12(P D G F R A)$ & $\begin{array}{l}\text { Forward: TCCAGTCACTGTGCTGCTTC } \\
\text { Reverse: GCAAGGGAAAAGGGAGTCTT }\end{array}$ & 260 \\
\hline $18(P D G F R A)$ & $\begin{array}{l}\text { Forward: ACCATGGATCAGCCAGTCTT } \\
\text { Reverse: TGAAGGAGGATGAGCCTGACC }\end{array}$ & 250 \\
\hline
\end{tabular}

Table II. Mutations in KIT and PDGFRA and the associations with the clinical and pathological features of GIST.

\begin{tabular}{|c|c|c|c|c|c|c|}
\hline \multirow[b]{2}{*}{ Parameter } & \multicolumn{2}{|c|}{$\operatorname{KIT}(\mathrm{n})$} & \multirow[b]{2}{*}{ P-value } & \multicolumn{2}{|c|}{$P D G F R A(\mathrm{n})$} & \multirow[b]{2}{*}{ P-value } \\
\hline & Mutant & No mutant & & Mutant & No mutant & \\
\hline Gender & & & 0.490 & & & 0.820 \\
\hline Male & 38 & 15 & & 3 & 50 & \\
\hline Female & 26 & 14 & & 1 & 39 & \\
\hline Age (years) & & & 0.260 & & & 1.000 \\
\hline$\geq 59$ & 23 & 14 & & 2 & 35 & \\
\hline$<59$ & 41 & 15 & & 2 & 54 & \\
\hline Diameter $(\mathrm{cm})$ & & & 0.446 & & & 0.683 \\
\hline$<5$ & 23 & 8 & & 1 & 30 & \\
\hline $5-10$ & 36 & 20 & & 3 & 53 & \\
\hline$>10$ & 5 & 1 & & 0 & 6 & \\
\hline Position & & & 0.040 & & & 0.002 \\
\hline Stomach & 53 & 18 & & 0 & 71 & \\
\hline Intestinal & 9 & 6 & & 2 & 13 & \\
\hline Outside the gastrointestinal tract & 2 & 5 & & 2 & 5 & \\
\hline Mitotic index (HPF) & & & 0.071 & & & 0.144 \\
\hline$<5 / 50$ & 23 & 6 & & 3 & 26 & \\
\hline $6-10 / 50$ & 33 & 14 & & 1 & 46 & \\
\hline$>10 / 50$ & 8 & 9 & & 0 & 17 & \\
\hline Risk classification & & & 0.057 & & & 0.086 \\
\hline Very low/low degree & 23 & 18 & & 0 & 41 & \\
\hline Moderate & 14 & 3 & & 1 & 16 & \\
\hline High & 27 & 8 & & 3 & 32 & \\
\hline
\end{tabular}

GIST, gastrointestinal stromal tumor; HPF, high-power fields.

male and 40 were female (male/female ratio, 1.33:1). The age range of the study population was 23-83 years (median age, 57 years), and the tumor diameter ranged between 5 and $45 \mathrm{~cm}$ (median diameter, $8.7 \mathrm{~cm}$ ). The tumors were distributed as follows: Gastric origin, 76.34\% (71/93); duodenum, $8.60 \%$ (8/93); ileum, $1.08 \%$ (1/93); colon, $1.08 \%$ (1/93); rectum,
5.38\% (5/93); esophagus, 5.38\% (5/93); pleural origin, $1.08 \%$ (1/93); and intrauterine origin, $1.08 \%$ (1/93). Based on the study by Fletcher et al (11), the risk of GIST was divided into three groups. Firstly, patients were classified with a very low risk of invasion if the tumor diameter was $<5 \mathrm{~cm}$ and they had a mitotic index of $<5 / 50$ high-power fields (HPF; 


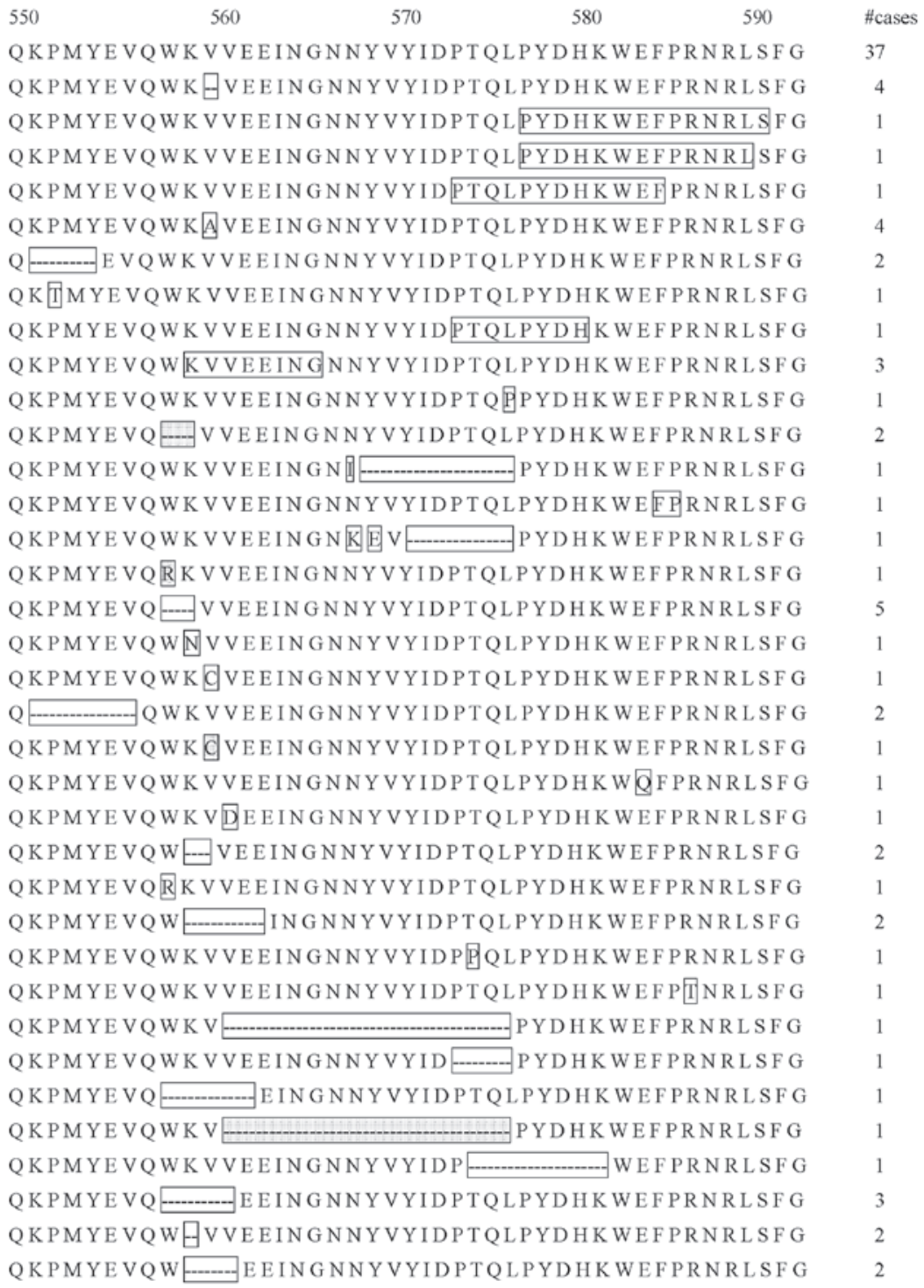

Figure 1. Schematic diagram showing the 37 without and 56 cases with a gastrointestinal stromal tumor that were shown to have mutations in exon 11 . White boxes represent a heterozygous deletion mutation. and gray boxes indicate homozygous deletion mutations. Boxes containing one or two letters represent heterozygous (if white) or homozygous (if gray) point mutations.

44.09\%, 41/93 samples). Secondly, patients were classified into the moderate risk invasion group if their tumor diameter ranged between 5 and $10 \mathrm{~cm}$ and had a mitotic index of $<5 / 50 \mathrm{HPF}$, or if a tumor with a diameter of $<5 \mathrm{~cm}$ was accompanied by a mitotic index of 6-10/50 HPF (18.28\%, $17 / 93$ samples). Finally, patients were classified with a high risk of invasion if they had a tumor diameter of $>5 \mathrm{~cm}$ and a mitotic index of $>5 / 50 \mathrm{HPF}$, a tumor diameter of $>10 \mathrm{~cm}$, or a mitotic index of $>10 / 50$ HPF (37.63\%, 35/93 samples). The CD117-positive cases accounted for $91.40 \%$ (85/93) of the study population, with the ages ranging between 23 and 83 years (median age, 58 years). The CD117-negative cases accounted for $8.60 \%$ (8/93) of the study population, with the ages ranging between 46 and 63 years (median age, 53 years). Of the eight CD117-negative cases, three were male and one was female, and two cases were of pleural origin, one case was of gastric origin, and one case was an esophageal tumor. These eight cases of GIST were followed-up and found to be malignant (Table II).
KIT mutation analysis. Among the 93 GIST cases, KIT mutations were detected in 64 cases $(68.82 \%)$, of which all were CD117-positive. Of the KIT mutations, $87.50 \%$ (56/64) were located on exon 11, 6.25\% (4/64) were located on exon 9, $4.69 \%(3 / 64)$ were located on exon 13 , which included a concomitant mutation on exon 11, and $1.56 \%(1 / 64)$ were located on exon 17, which included a concomitant mutation of exon 11. In the CD117-positive cases, the mutation ratio of KIT reached $75.29 \%$ (64/85). Among the mutations on exon $11,91.07 \%$ (51/56) were heterozygous and only $8.93 \%$ (5/56) were homozygous. As shown in Fig. 1, the mutation types identified on exon 11 included deletion mutations $(55.36 \%, 31 / 56)$, point mutations $(26.79 \%, 15 / 56)$, insertion mutations (tandem repeats; $14.29 \%, 8 / 56$ ) and point mutations in the deletion mutation $(3.57 \%, 2 / 56)$. The majority of the mutations $(62.50 \%, 35 / 56)$ were located at the 'hot' zone, involving codons 550-560, at the 5'-end. The most common form was the WK (Trp-Lys) deletion mutation at the 5'-end of codons 557-558 $(12.50 \%, 7 / 56)$, followed by the 
A

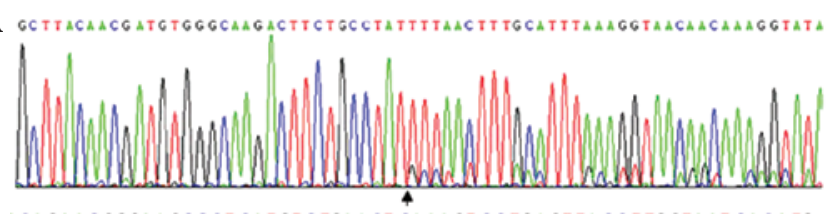

B

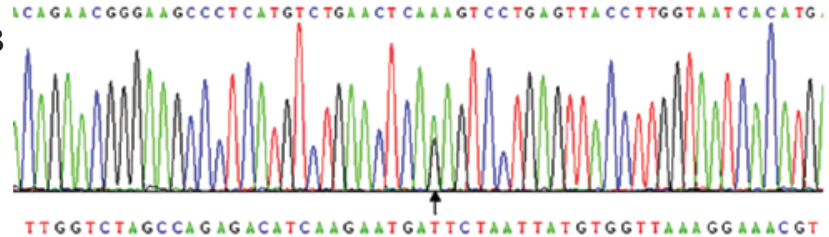

C
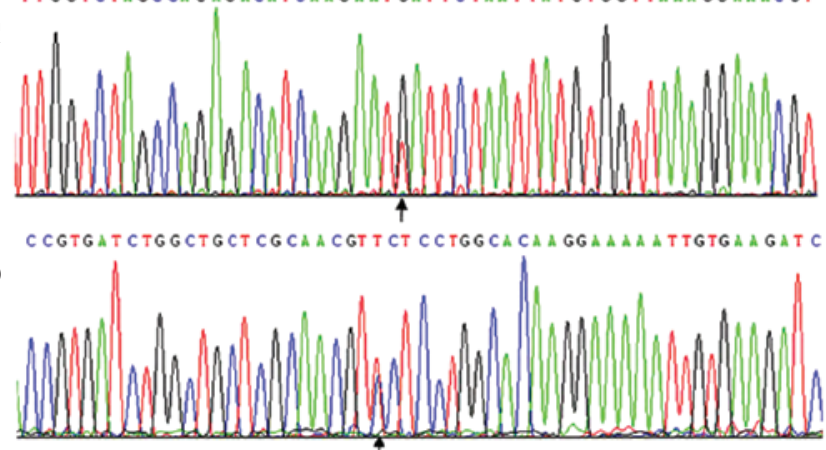

Figure 2. Sequence diagram showing the point mutations in the gastrointestinal stromal tumors. (A) Mutations detected in exon 9 of KIT corresponded to codons 502 and 503 (Ala-Tyr) and comprised six internal tandem duplications, which were heterozygous mutations. (B) Mutations detected in exon 13 of $K I T$ corresponded to a K642R point mutation. (C) Mutations detected in exon 17 of $K I T$ corresponded to a D820Y point mutation. (D) Mutations detected in exon 18 of PDGFRA corresponded to Val 824 internal GTC $>$ GTT base point mutations.

$\mathrm{V}$ (Val) deletion mutation at codon 559, and the V575A point mutation $(7.14 \%, 4 / 56)$. In $14.29 \%(8 / 56)$ of the GIST cases, 2-14 amino acids were inserted into exon 11, including five cases with 3 '-end tandem repeats of internal tandem duplications (ITDs) and three cases with 5'-end ITDs. Among the five cases with the 3 '-end ITDs, two were female and three were male. In $6.25 \%$ (4/64) of the GIST cases, $K I T$ mutations were detected in exon 9, which corresponded to codons 502 and 503 (Ala-Tyr) with six ITDs, of which three were heterozygous mutations (Fig. 2A). Three cases originated in the duodenum and one case originated in the stomach. In $4.69 \%(3 / 64)$ of the GIST cases, mutations were detected in exon 13, which comprised a K642R point mutation (Fig. 2B). Only in $1.56 \%$ (1/64) of the GIST cases was a mutation detected in exon 17 , and this mutation coexisted with a double deletion mutation on exon 11 at codons 551-554 (KPMY, Lys-Pro-Met-Tyr) and with a D820Y point mutation (Fig. 2C).

PDGFRA mutation analysis. Among the 29 GIST cases without a KIT mutation, a mutation in PDGFRA was detected in three cases $(3.23 \%, 3 / 93 ; 10.34 \%, 3 / 29)$. Only one GIST patient with a mutation in PDGFRA on exon 18, which corresponded to a Val 824 internal GTC $>$ GTT base point mutation, also had a mutation in exon 11 of KIT, which corresponded to a L576P point mutation (Fig. 2D). The remaining three cases also exhibited mutations on exon 18 , all of which corresponded to a $\mathrm{D} 842 \mathrm{~V}$ point mutation. These three patients with a mutation in PDGFRA were CD117-negative.

\section{Discussion}

The c-Kit proto-oncogene is located on the long arm of the fourth human chromosome, within zones 2 and 3 (4q12-13). The product of KIT is a type III tyrosine kinase growth factor receptor, which is a $145-\mathrm{kDa}$ transmembrane glycoprotein. The KIT gene contains 21 exons, and activation of the gene depends on ligand binding with stem cell factor, which enables the phosphorylation of substrate proteins. Subsequently, certain signal transduction pathways are activated, which stimulate important cellular functions, such as proliferation and apoptosis. Mutations in KIT that cause autophosphorylation without the presence of the ligand lead to uncontrolled cell proliferation, which eventually induces tumor development (12). Furthermore, Hirota et al (12) indicated that a gain-of-function mutation within KIT may be one of the core events underlying tumor development, with the localization of the intracellular membrane-proximal domain of the c-Kit protein (mutation on exon 11) being the most common mutation. Due to the differences in the tissues used for DNA extraction, the experimental methods employed and the cases constituting each study group, previous studies have reported a wide range of mutation rates, varying between 21 and 92\% (9,13-15). Three quantitative studies conducted using a large study population from other countries revealed that the mutation rate of exon 11 in GISTs was 69.32\% (296/427), 72.36\% (233/322) and 51.50\% $(103 / 200)(15-17)$, respectively, which is consistent with the results of the present study $(60.21 \%, 56 / 93)$.

Analysis of the clinicopathological features of GIST in the present study revealed that mutations in KIT were the only significant variable $(\mathrm{P}=0.040)$, while gender, age, tumor diameter, mitotic index and risk classification exhibited no statistically significant differences $(\mathrm{P}>0.05)$. The primary location of the KIT mutations was at the 5 '-end of exon 11 , with point mutations and in-frame deletions being the most relevant events, whereas the second most common location was at the 3 '-end of the same exon, in which ITDs were the predominant events. In accordance with the results of the present study, previous studies have demonstrated that the latter type of mutation occurs primarily in the stomach and is more common in female patients $(18,19)$. In the current study, only four cases were identified to have a mutation in exon 9 of $K I T$, which corresponded to the extracellular domain of c-Kit. This mutation rate was similar to that reported by Lasota et al who used a larger cohort of samples $(3.00 \%, 6 / 200)(17)$, but slightly lower compared with the 7.2-10.84\% reported by Corless et al (16), Antonescu et al (18) and Wozniak et al (15). Mutations on exon 17 of KIT, which correspond to the phosphotransferase domain of c-Kit, have been rarely observed. In the present study, one case was found to have an exon 17 mutation that coexisted with a D820Y point mutation on exon 11, which was newly identified. Although the identification of genetic mutations is an important tool, analysis of CD117-negative GIST mutations is very difficult, and the results reported in the literature are not consistent $(20,21)$.

PDGFRA and KIT are located on adjacent positions on the fourth human chromosome and share high amino acid sequence homology (1). Heinrich et al (1) found that in cases of GIST without KIT mutations, PDGFRA mutations were more frequent, which may be one of the mechanisms underlying 
GIST development. The PDGFRA gene, whose signal transduction pathway is similar to that of KIT, contains 23 exons and has an epithelial cell-based phenotype. Mutations in PDGFRA can generate autophosphorylation, which triggers a signal transduction cascade that enables uncontrolled and disordered cell growth (1). Analysis of the clinicopathological factors of GIST in the present study indicated that mutations in KIT were the only significant events $(\mathrm{P}=0.002)$, while gender, age, tumor diameter, mitotic index and risk classification exhibited no statistically significant differences $(\mathrm{P}>0.05)$. In the present study, among the 93 samples studied, mutations in PDGFRA accounted for $5.30 \%$ (4/93) of the sample population, with one case coexisting with a mutation on exon 11 of KIT. The remaining three cases $(10.34 \%, 3 / 29)$ were represented by a D842V point mutation on exon 18 , associated with a lack of KIT expression, which was observed in $37.50 \%$ (3/8) of the CD117-negative cases. Tu et al (22) reported the case of a renal transplant recipient with a GIST, in which a Val824 internal GTC $>$ GTT base point mutation was identified in PDGFRA with no KIT mutations present. This was the second report of this type of mutation, which coexisted with the L576P point mutation on exon 11.

Imatinib, which exerts a selective inhibition on $B C R-A B L$, $P D G F R A$ and $K I T$, is a small molecular agent with tyrosine kinase activity whose clinical treatment for GIST is very effective (23). However, the location and nature of the mutations can affect the response of GIST to imatinib (24). A previous study found that GIST patients with a mutation on exon 11 of KIT exhibited a good response to imatinib, while the response of patients with a mutation on exon 9 to Gleevec was slightly worse (25). A number of in vitro and clinical studies have indicated that for cases with mutations in the kinase locus, such as mutations in exon 17 of KIT or in exon 18 of PDGFRA, treatment with the inhibitor should have been terminated (26). However, additional studies have demonstrated that $30 \%$ of the mutations in PDGFRA are sensitive to imatinib, indicating that attention should be focused on CD117-negative GIST cases, and that the corresponding genetic mutations should be tested against imatinib (27).

In conclusion, to the best of our knowledge, the present study is the first to investigate the associations between mutations in the KIT and PDGFRA genes with GIST in a cohort of patients from North China. The results of the present study demonstrated that the most frequent mutation occurred on the two ends of exon 11 in KIT. In addition, mutations in PDGFRA were primarily observed in CD117-negative GIST patients. Notably, one case was identified to have a syngeneic double mutant on exon 17 of PDGFRA and exon 11 of $K I T$, which to the best of our knowledge, is the only case to have been detected worldwide. To date, only one case of an allogeneic double mutant has been reported in China, which involved a Val824 internal GTC $>$ GTT base point mutation on exon 18 of PDGFRA. In the present study, gene mutation types were identified using PCR amplification and sequencing method, and associated gene mutations were analyzed to determine the development process, prognosis and impact of imatinib-targeted therapy in patients with GIST, indicating that it may provide a molecular basis for evaluating pathologies.

\section{References}

1. Heinrich MC, Corless CL, Duensing A, McGreevey L, Chen CJ, Joseph N, Singer S, Griffith DJ, Haley A, Town A, et al: PDGFRA activating mutations in gastrointestinal stromal tumors. Science 299: 708-710, 2003.

2. Nedeljkovic SS, Wasan A and Jamison RN: Assessment of efficacy of long-term opioid therapy in pain patients with substance abuse potential. Clin J Pain 18 (4 Suppl): S39-S51, 2002.

3. Joensuu H and Kindblom LG: Gastrointestinal stromal tumors - a review. Acta Orthop Scand Suppl 75: 62-71, 2004.

4. Lasota J, Jasinski M, Sarlomo-Rikala M and Miettinen M: Mutations in exon 11 of c-kit occur preferentially in malignant versus benign gastrointestinal stromal tumors and do not occur in leiomyomas or leiomyosarcomas. Am J Pathol 154: 53-60, 1999.

5. Kim TW, Lee H, Kang YK, Choe MS, Ryu MH, Chang HM, Kim JS, Yook JH, Kim BS and Lee JS: Prognostic significance of c-kit mutation in localized gastrointestinal stromal tumors. Clin Cancer Res 10: 3076-3081, 2004.

6. Corless CL, McGreevey L, Haley A, Town A and Heinrich MC: KIT mutations are common in incidental gastrointestinal stromal tumors one centimeter or less in size. Am J Pathol 160: 1567-1572, 2002.

7. He HY, Fang WG, Zhong HH, Li Y, Zheng J, Du J, Heng WJ and $\mathrm{Wu} B Q$ : Status and clinical implication of c-kit and PDGFRA mutations in 165 cases of gastrointestinal stromal tumor (GIST). Zhonghua Bing Li Xue Za Zhi 35: 262-266, 2006 (In Chinese).

8. Debiec-Rychter M, Dumez H, Judson I, Wasag B, Verweij J Brown M, Dimitrijevic S, Sciot R, Stul M, Vranck H, Scurr M, Hagemeijer A, van Glabbeke $M$ and van Oosterom AT; EORTC Soft Tissue and Bone Sarcoma Group: Use of c-KIT/PDGFRA mutational analysis to predict the clinical response to imatinib in patients with advanced gastrointestinal stromal tumours entered on phase I and II studies of the EORTC soft tissue and bone sarcoma group. Eur J Cancer 40: 689-695, 2004.

9. Miettinen M and Lasota J: Gastrointestinal stromal tumors: Review on morphology, molecular pathology, prognosis and differential diagnosis. Arch Pathol Lab Med 130: 1466-1478, 2006.

10. Piao J, Liu S, Xu Y, Wang C, Lin Z, Qin Y and Liu S: Ezrin protein overexpression predicts the poor prognosis of pancreatic ductal adenocarcinomas. Exp Mol Pathol 98: 1-6, 2015.

11. Fletcher CD, Berman JJ, Corless C, Gorstein F, Lasota J, Longley BJ, Miettinen M, O'Leary TJ, Remotti H, Rubin BP, et al: Diagnosis of gastrointestinal stromal tumors: A consensus approach. Hum Pathol 33: 459-465, 2002.

12. Hirota S, Isozaki K, Moriyama Y, Hashimoto K, Nishida T, Ishiguro S, Kawano K, Hanada M, Kurata A , Takeda M, et al: Gain-of-function mutations of c-kit in human gastrointestinal stromal tumors. Science 279: 577-580, 1998.

13. Hou YY and Zhu XZ: Gastrointestinal stromal tumor molecular biology. Cancer Res Clin 18: 507-512, 2006.

14. Battochio A, Mohammed S, Winthrop D, Lefresne S, Mulder K, Chu Q, O'Hara C and Lai R: Detection of c-KIT and PDGFRA gene mutations in gastrointestinal stromal tumors: Comparison of DHPLC and DNA sequencing methods using a single population-based cohort. Am J Clin Pathol 133: 149-155, 2010.

15. Wozniak A, Rutkowski P, Piskorz A, Ciwoniuk M, Osuch C, Bylina E, Sygut J, Chosia M, Rys J, Urbanczyk K, et al; Polish Clinical GIST Registry: Prognostic value of KIT/PDGFRA mutations in gastrointestinal stromal tumours (GIST): Polish Clinical GIST Registry Experience. Ann Oncol 23: 353-360, 2012.

16. Corless CL, Fletcher JA and Heinrich MC: Biology of gastrointestinal stromal tumors. J Clin Oncol 22: 3813-3825, 2004.

17. Lasota J, Wozniak A, Sarlomo-Rikala M, Rys J, Kordek R, Nassar A, Sobin LH and Miettinen M: Mutations in exons 9 and 13 of KIT gene are rare events in gastrointestinal stromal tumors. A study of 200 cases. Am J Pathol 157: 1091-1095, 2000.

18. Antonescu CR, Sommer G, Sarran L, Tschernyavsky SJ, Riedel E, Woodruff JM, Robson M, Maki R, Brennan MF, Ladanyi M, et al: Association of KIT exon 9 mutations with nongastric primary site and aggressive behavior: KIT mutation analysis and clinical correlates of 120 gastrointestinal stromal tumors. Clin Cancer Res 9: 3329-3337, 2003. 
19. Lasota J, Dansonka-Mieszkowska A, Stachura T, Schneider-Stock R, Kallajoki M, Steigen SE, Sarlomo-Rikala M, Boltze C, Kordek R, Roessner A, et al: Gastrointestinal stromal tumors with internal tandem duplications in 3 ' end of KIT juxtamembrane domain occur predominantly in stomach and generally seem to have a favorable course. Mod Pathol 16: 1257-1264, 2003

20. Medeiros F, Corless CL, Duensing A, Hornick JL, Oliveira AM, Heinrich MC, Fletcher JA and Fletcher CD: KIT-negative gastrointestinal stromal tumors: Proof of concept and therapeutic implications. Am J Surg Pathol 28: 889-894, 2004.

21. Tzen CY and Mau BL: Analysis of CD117-negative gastrointestinal stromal tumors. World J Gastroenterol 11: 1052-1055, 2005.

22. Tu H, Li Q, Cai J, Chen Z, Yang H, Jiang H, Mao Y, Shou Z and Chen J: Extragastrointestinal stromal tumor in a kidney transplant recipient. Clin Exp Nephrol 16: 350-353, 2012.
23. Li J: Molecular target therapy for gastrointestinal stromal tumor. Zhong Hua Xiao Hua Wai Ke Za Zhi 12: 253-256, 2013 (In Chinese).

24. Nickl NJ: Gastrointestinal stromal tumors: New progress, new questions. Curr Opin Gastroenterol 20: 482-487, 2004.

25. Chen H: Mutation of c-kit and PDGFRA Genes in Gastrointestinal Stromal Tumor. Zhong Guo Sheng Wu Hua Xue Yu Fen Zi Sheng Wu Xue Bao 12: 697-701, 2009 (In Chinese).

26. He H, Xiang Y, Li Y, Zhong G, Wu B and Zheng J. c-kit and PDGFRA mutat ions in 60 cases of gastrointestinal stromal tumors (GISTs). Beijing Da Xue Xue Bao 37: 320-324, 2005 (In Chinese)

27. Kim S Y, Janeway K and Pappo A: Pediatric and wild-type gastrointestinal stromal tumor: New therapeutic approaches. Curr Opin Oncol 22: 347-350, 2010. 